\title{
ON THE GENERATION OF ENHANCED LOOKUP TABLES FOR WHEEL-RAIL CONTACT MODELS
}

\author{
F. Marques ${ }^{1, *}$, H. Magalhães ${ }^{2}$, B. Liu ${ }^{3}$, J. Pombo ${ }^{2,4}$, P. Flores ${ }^{1}$, J. Ambrósio ${ }^{2}$, \\ J. Piotrowski ${ }^{5}$, S. Bruni ${ }^{3}$ \\ ${ }^{1}$ CMEMS-UMinho, Departamento de Engenharia Mecânica, Universidade do Minho, \\ Campus de Azurém, 4804-533 Guimarães, Portugal \\ ${ }^{2}$ LAETA, IDMEC, Instituto Superior Técnico, Universidade de Lisboa, \\ Avenida Rovisco Pais 1, 1049-001 Lisboa, Portugal \\ ${ }^{3}$ Dipartimento di Meccanica, Politecnico di Milano Via La Masa 1, Milano 20156, Italy \\ ${ }^{4}$ Institute of Railway Research, School of Computing and Engineering, \\ University of Huddersfield, UK and ISEL, IPL, Lisboa, Portugal \\ ${ }^{5}$ Institute of Vehicles, Warsaw University of Technology Narbutta 84, 02-524 Warsaw, Poland
}

\begin{abstract}
In railway dynamics, the interpolation of lookup tables (LUTs) is a procedure to reduce the computational effort when computing the wheel-rail interaction forces. However, the generation of LUTs with multiple inputs and multiple outputs is a challenging task for which issues such as their minimal size and uniform accuracy over the LUT domain have not been systematically addressed before. This work presents a comprehensive methodology for a detailed analysis of general LUTs, identifying ways to improve them. First, an analysis of the variation of the input parameters is made and the interpolation error is assessed on the cells and edges of the original table. From this analysis, two enhanced LUTs are proposed. One is approximately 5 times smaller than the original but holds similar accuracy. The other table exhibits half of the maximum interpolation error of the original LUT but holds an identical size. This methodology is demonstrated here using the recently published Kalker Book of Tables for Non-Hertzian contact (KBTNH) but it can be used by any other LUT approach in order to improve accuracy and/or to reduce size.
\end{abstract}

Keywords: Wheel-Rail Contact; Lookup Tables; Accuracy; Efficiency; Non-Hertzian Contact;

\section{Introduction}

The wheel-rail contact interaction representation plays a fundamental role in the study and simulation of railway vehicles [1], namely in vehicle dynamics [2-5], prediction of wear or rolling contact fatigue [6-8], traction and braking systems design [9,10], among others. Hence, the main focus of research works has been the development of more advanced contact models to enhance the existing algorithms either in terms of accuracy or efficiency [11-16]. It has been demonstrated that the selected methodology for the evaluation of creep forces has paramount influence on the dynamic response of the vehicle [17]. The ultimate goal of developing a new contact model is to provide high accuracy by capturing most of tribological phenomena while keeping a low computation effort. More advanced nonlinear models [11,18-20] are required for situations where large creepages or conformal contact exist, for instance, and to study contact damage phenomena, such as wear or rolling contact fatigue. In opposition, simplified linear models [21-23] are more suitable when the local contact phenomena are not particularly relevant. These models can also have special interest for real-time simulations or other cases in which the computational efficiency is a key aspect. In this framework, although

\footnotetext{
*E-mail: fmarques@ dem.uminho.pt
} 
it is not the standard procedure, the utilization of LUT based approaches is a valuable and common alternative for efficiency improvement with respect to online contact force evaluation.

The use of LUT in the wheel-rail contact requires the generation of a pre-calculated table which stores the values of the function, such as the normal and creep forces, in a set of points that constitute the domain of the table. Then, during a simulation, for a given input, such as the relative position and orientation between rail and wheel, the output parameters required for the dynamic analysis are computed through the interpolation of the LUT. Thus, the online calculation of a cumbersome function, which is generally slow but accurate, can be promptly approximated through the linear interpolation of tabulated data.

LUT approaches are employed in a wide variety of fields in which the computational efficiency takes a preponderant role [24-26]. Bearing in mind that the dynamic simulations of railway vehicles usually involve high computational costs, the contact detection [27,28] and the contact forces evaluation [29,30] are frequently performed recurring to LUT approaches. Regarding wheel-rail contact forces, the first LUT was developed by the British Rail [31] for a parametrized elliptical contact patch where Kalker's DUVOROL program was utilized to calculate the creep forces [18]. Since the LUT was constrained to Hertzian contact patches, the elliptical regularization of the non-elliptical contact patches was required. This table had four input parameters and a relatively small size with a total of 3220 entries as, at the time, the pre-calculation time and the computer storage had limitations. Later, Kalker created a larger LUT called USETAB [29], also based on elliptical regularization of the contact patch. The table had 115000 entries in which the stored creep forces were determined with the CONTACT software [11]. This LUT was enhanced in [32] resulting in a table of 100000 entries called USETAB 1.2. This improved version is currently used in commercial software, namely NUCARS and VAMPIRE. More recently, a new Kalker Book of Tables for NonHertzian contact (KBTNH), in which a non-elliptical contact patch regularization has been suggested using five input parameters, was introduced by Piotrowski et al. [30,33]. This regularization assumes that the contact patch can be approximated by a single double elliptical contact shape, which allows to capture the longitudinal creep force generated by the spin creepage.

Vollebregt et al [34] demonstrated that the USETAB provides significantly better results than other simplified approaches [21-23] when compared with the complete rolling contact theory [11]. Note that the accuracy of the interpolated values must be controlled, since the function to be replaced is already an approximation of the reality. Thus, the design of the LUT domain is of paramount importance due to its direct influence in the error obtained. Although the increase of the number of points of the LUT tends to improve its accuracy, it also increases the size of the table, the effort required to generate it, and the computation time needed to search and interpolate a given point during the dynamic analysis. Therefore, accuracy and efficiency issues make the selection of the number of LUT points and their location in the complete domain of extreme importance. Typically, this selection is made empirically, since there is a lack of systematic techniques either to evaluate the LUT accuracy or to generate it. Most of the existing methods are constrained to single input and output LUTs [35-37] and cannot be extended to multiple inputs and outputs LUTs [38].

The main objective of the current work is to analyze the error over a LUT domain and to propose a methodology to generate enhanced LUTs either with smaller sizes or higher accuracies. This approach is applied and demonstrated using the recently published KBTNH [30,33]. The remainder of the paper is organized as follows: Section 2 describes 
briefly the KBTNH parametrization, as well as the original domain distribution; The assessment of the LUT accuracy through a sensitivity analysis and evaluation of the interpolation error in the cells and edges of the LUT is presented in Section 3; Then, two enhanced versions of the KBTNH are proposed in Section 4; Finally, the main conclusions of this work are drawn in Section 5.

\section{The Kalker Book of Tables}

The contact between the rail and the wheel tread, in normal operating conditions with regular wheel and rail profiles, is characterized by a configuration such as that illustrated in Fig. 1.

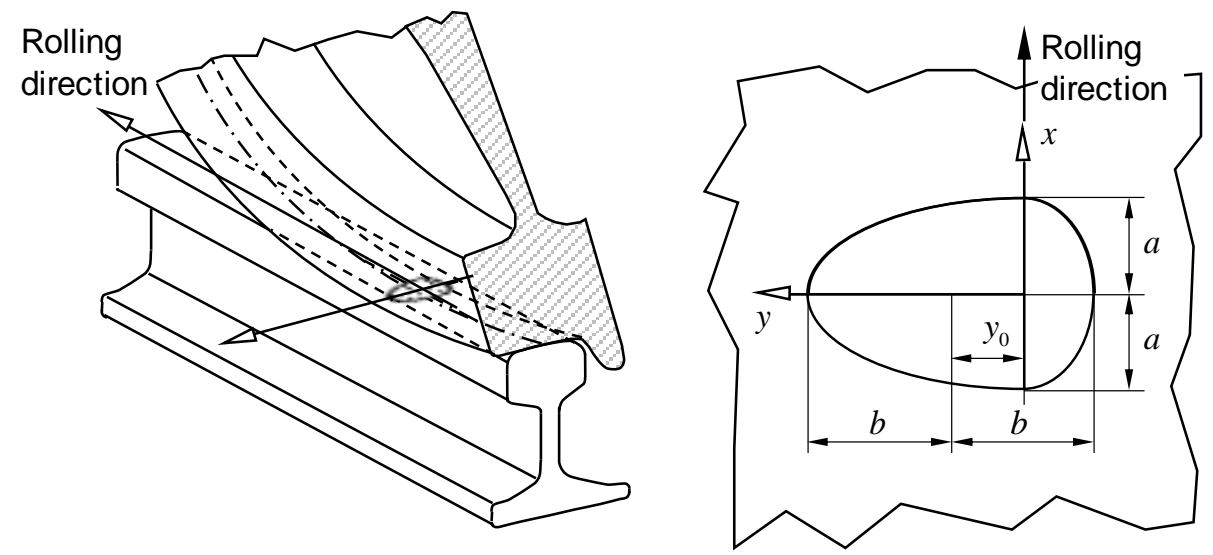

Figure 1 Representation of the wheel-rail contact and Simple Double-Elliptical Contact region (SDEC).

A regularization of non-elliptical contact patches has been introduced by Piotrowski et al. [30,33], being the contact patch regularized to be a Simple Double-Elliptical Contact region (SDEC), as represented in Fig. 1. This regularization allows to build a LUT based on CONTACT software [11] for the creep forces and moment calculation, designated as the Kalker Book of Tables for Non-Hertzian contact [30,33]. Consider that the SDEC region is suitable to regularize most of the contact shapes obtained with approximate nonHertzian methods for normal contact problem. Notice that the SDEC shape holds some quasi-Hertzian properties and symmetry relations, and it requires a small number of parameters to be fully described. Hence, in a nondimensionalized analysis, it can be characterized by the semi-axes ratio as

$$
g=a / b
$$

and the shape number as

$$
\psi=y_{0} / b
$$

where $a$ and $b$ are the semi-axes of the SDEC region. For a given pair of surfaces, recurring to the parametrization of variables, the creep forces and moment generated due to wheel-rail interaction only depend on the contact patch geometry, characterized by $g$ and $\psi$, and the parametrized longitudinal, lateral and spin creepages. Moreover, the parametrized creepages, designated by $\xi, \eta$ and $\chi$ respectively, are defined as

$$
\xi=\frac{\rho v_{x}}{\mu c} \quad, \quad \eta=\frac{\rho v_{y}}{\mu c} \quad, \quad \chi=\frac{\rho \varphi}{\mu}
$$

where $\rho$ is a characteristic length of the elliptical contact patch, $\mu$ denotes the friction coefficient, $v_{x}$ represents the longitudinal creepage, $v_{y}$ is the lateral creepage, $\varphi$ denotes the spin creepage, and $c$ is the equivalent radius of the SDEC region which can be calculated as

$$
c=\sqrt{a b}
$$

Moreover, the characteristic length of the elliptical contact patch can be evaluated as 


$$
\rho=\frac{4 E}{3\left(1-\sigma^{2}\right) N}\left(\frac{A}{\pi n_{a} n_{b}}\right)^{\frac{3}{2}}
$$

in which $E$ represents the Young's modulus, $\sigma$ denotes the Poisson ratio, $A$ is the area of the contact patch, $N$ represents the normal contact force, and $n_{\mathrm{a}}$ and $n_{\mathrm{b}}$ are tabulated coefficients which are dependent of $\theta$, which is given

$$
\theta=\max \left(\frac{a}{b}, \frac{b}{a}\right)
$$

Hence, the product of these tabulated coefficients can be approximated by the following empirical formula

$$
n_{a} n_{b}=k_{3} \theta^{3}+k_{2} \theta^{2}+k_{1} \theta+k_{0}
$$

where $k_{3}=3.618652 \times 10^{-5}, k_{2}=-2.687505 \times 10^{-3}, k_{1}=9.5658 \times 10^{-2}$ and $k_{0}=8.872736 \times 10^{-1}$.

For the sake of simplicity, the regularized longitudinal and lateral creepages are replaced by the creepage angle and modulus of creepage, which are defined as

$$
v=\sqrt{\xi^{2}+\eta^{2}} \quad, \quad \alpha=\tan ^{-1}\left(\frac{\eta}{\xi}\right)
$$

For this case, the generation of the lookup table involves five input variables, each one with a characteristic domain:

$\chi \in[0,2]$ - parametrized spin creepage;

$g \in[0.2,5]$ - semi-axes ratio;

$\alpha \in[-\pi / 2, \pi / 2]$ - creepage angle;

$v \in[0,12]$ - parametrized creepage modulus;

$\psi \in[-0.9,0.9]$ - shape number;

and three output quantities:

$f_{x}$ - normalized longitudinal creep force;

$f_{y}$ - normalized lateral creep force;

$m_{z}$ - normalized moment.

The normalized creep forces and moment are obtained from the contact loads calculated with CONTACT as

$$
\begin{aligned}
f_{x}(\chi, g, \alpha, v, \psi) & =\frac{F_{x}}{\mu N} \\
f_{y}(\chi, g, \alpha, v, \psi) & =\frac{F_{y}}{\mu N} \\
m_{z}(\chi, g, \alpha, v, \psi) & =\frac{M_{z}}{\mu c N}
\end{aligned}
$$

where $F_{x}, F_{y}$ and $M_{z}$ are, respectively, the longitudinal and lateral creep forces and the moment computed by CONTACT, and.

A reasonable LUT has been built which spans the complete domain in which the expected contact scenarios for typical vehicle-track interaction are included. This justifies the chosen limits for the spin creepage and semi-axes ratio. Furthermore, it should be noted that the domain of the longitudinal and spin creepages is reduced to positive values due to symmetry relations provided by the SDEC shape [30], thus decreasing significantly the size of the table.

Table 1 shows the distribution of points of the LUT proposed by Piotrowski et al. [30]. As can be observed, a different number of points was utilized for the several independent parameters. In this case, the selection of the number and position of the LUT points has been done empirically, recurring to the users' experience. The most refined parameter is by far the parametrized creepage modulus. Thus, the total number of LUT 
points, and runs of program CONTACT required to compute the forces, is calculated as the combination of these points

$$
N_{\mathrm{p}}=\prod_{i=1}^{5} n_{i}=113344
$$

This number represents the LUT size and it has direct impact on the storage size and on the time required to search the table. It seems obvious that it is of great interest to keep the number of LUT points as low as possible.

Thus, the evaluation of the creep forces and moment during a multibody simulation is made through the assessment of the LUT values and subsequently linear interpolation in the five-dimensional space.

Table 1 Domain of the Kalker Book of Tables for Non-Hertzian (KBTNH) contact.

\begin{tabular}{|c|c|}
\hline Parameter & No. of Points \\
\hline$\chi=\left\{\begin{array}{llll}0.00 & 0.25 & 0.50 & 1.00 \\
1.25 & 1.50 & 1.75 & 2.00\end{array}\right\}$ & $n_{\chi}=8$ \\
\hline$g=\left\{\begin{array}{llllllll}\frac{1}{5} & \frac{1}{2} & \frac{3}{4} & 1 & 2 & 3 & 4 & 5\end{array}\right\}$ & $n_{\mathrm{g}}=8$ \\
\hline$\alpha=\left\{\begin{array}{rrrrrrr}-\frac{\pi}{2} & -\frac{\pi}{3} & -\frac{\pi}{6} & 0 & \frac{\pi}{6} & \frac{\pi}{3} & \frac{\pi}{2}\end{array}\right\}$ & $n_{\alpha}=7$ \\
\hline 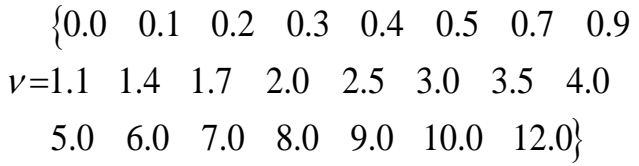 & $n_{v}=23$ \\
\hline$\psi=\left\{\begin{array}{llllll}-0.9 & -0.8 & -0.6 & -0.4 & -0.2 \\
0.0 & 0.2 & 0.4 & 0.6 & 0.8 & 0.9\end{array}\right\}$ & $n_{\psi}=11$ \\
\hline
\end{tabular}

\section{Assessment of the Lookup Table Accuracy}

A comprehensive evaluation of the LUT accuracy is performed here by analyzing the variation of its input parameters and quantifying how each parameter affects the calculation of the creep forces. First, a mesh of 60x60 elements is utilized in CONTACT to discretize the complete contact patch. Then, the assessment of the interpolation error is performed by comparing the exact value of the contact force evaluated with CONTACT and the approximated value obtained with linear interpolation of LUT data. The center point of each cell and the middle point of each edge are used to evaluate this error. Note that by point it is meant a set of values of input parameters.

\subsection{Analysis of the Variation of the Input Parameters}

The variation of each output parameter, i.e., the two force components and moment, to each one of the input parameters is evaluated in order to understand which are the parameters that need a finer or coarser discretization. Bearing this in mind, with a design of experiments technique [39], ten points inside the domain of the lookup table are selected with a Latin Hypercube Sampling (LHS) method, as listed in Table 2. This sampling technique allows a suitable inspection of the domain. 

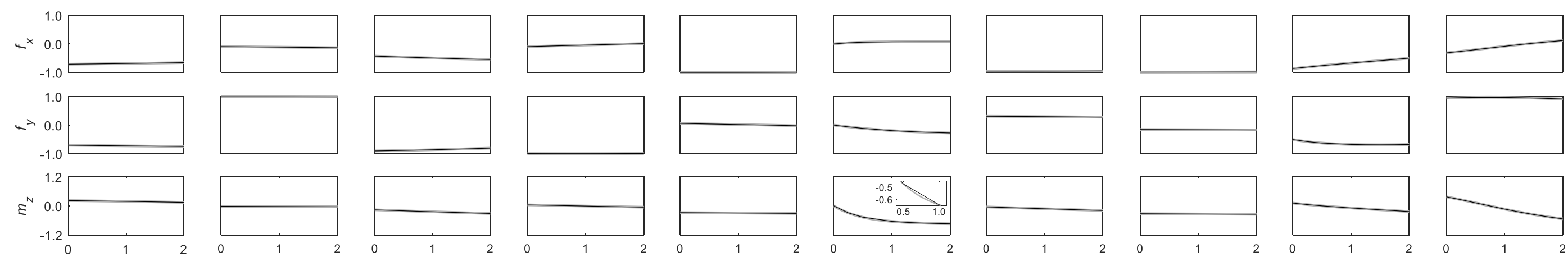

Parametrized spin creepage $(\chi)$
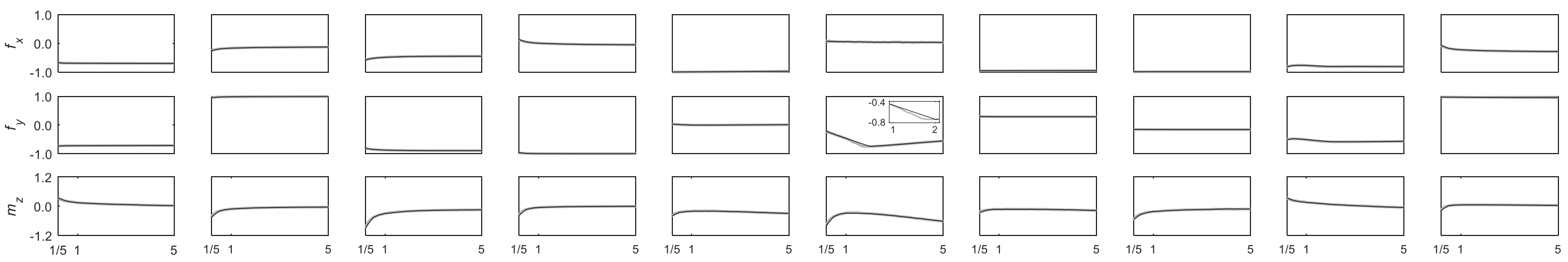

Semi-axes ratio $(g)$
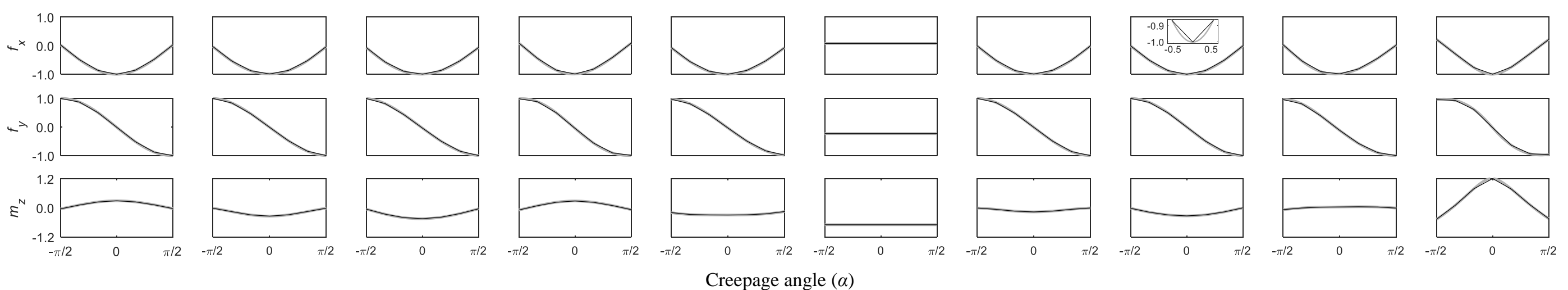

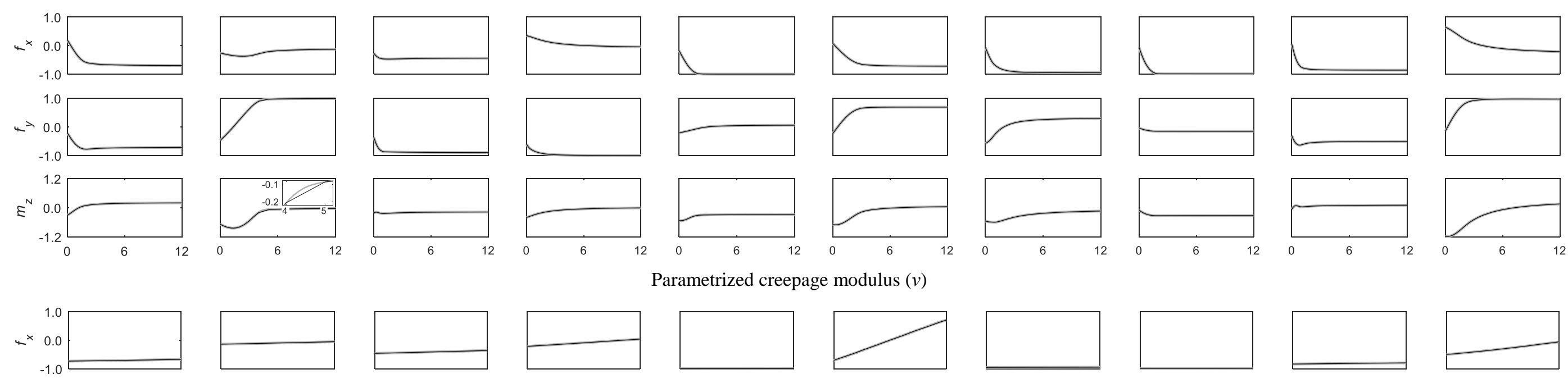

Parametrized creepage modulus $(v)$
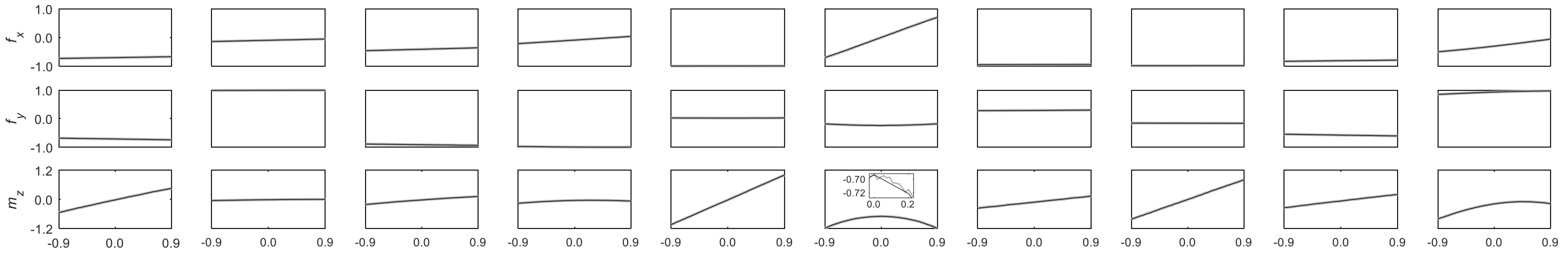

Figure 2 Analysis of the variation of the output quantities of the function used to generate the KBTNH using a LHS with 10 points listed in Table 2 ( - solid thick grey line - results for 100 values of each input parameter; - solid thin black line - results for the LUT values) 
Table 2 Points of the Lookup Table selected using a Latin Hypercube Sampling.

\begin{tabular}{|c|c|c|c|c|c|}
\hline Point & $\chi$ & $g$ & $\alpha$ & $v$ & $\psi$ \\
\hline 1 & 0.76 & 0.63 & 0.79 & 9.00 & 0.378 \\
\hline 2 & 1.88 & 4.52 & -1.48 & 11.04 & -0.702 \\
\hline 3 & 0.50 & 1.90 & 1.13 & 3.12 & -0.900 \\
\hline 4 & 1.72 & 1.18 & 1.48 & 6.60 & 0.612 \\
\hline 5 & 0.96 & 0.32 & -0.06 & 3.84 & -0.252 \\
\hline 6 & 1.36 & 0.24 & -0.75 & 0.00 & 0.090 \\
\hline 7 & 1.48 & 4.20 & -0.31 & 10.20 & -0.162 \\
\hline 8 & 0.14 & 0.49 & 0.16 & 7.32 & -0.360 \\
\hline 9 & 0.28 & 2.61 & 0.53 & 1.44 & 0.342 \\
\hline 10 & 1.02 & 0.21 & -1.26 & 5.28 & 0.828 \\
\hline
\end{tabular}

The sensitivity of the output quantities, i.e., the three forces to each one of the five input parameters are calculated about each one of the ten sampling points by varying one of the input parameters while maintaining the other four unchanged. Thus, Figure 2 presents the results of this variation in which each parameter is tested for one hundred values within its domain. The variation of the input parameters for the LUT values is also included for sake of comparison. Since both lines seem almost overlapping in the full scale, a representative plot of each input parameter was amplified in order to observe the existence of the error caused by the linear interpolation.

The analysis of the variation of the outputs shown in Fig. 2 provides useful indications on the requirements for finer or coarser discretization. The spin creepage shows a more pronounced nonlinear behavior in the left side of the domain being almost linear in the right side. The semi-axes ratio seems to have a logarithmic like behavior, which suggests having an equivalent refinement from $1 / 5$ to 1 and from 1 to 5 . The creepage angle leads to a force and moment response that resembles a sinusoidal behavior. It is observed that the creepage modulus is highly nonlinear for low values, which suggests refinement, becoming almost linear for high values of creepage. The shape number leads to a very moderate nonlinear behavior, thus, a small number of points is required to provide a good approximation. It is also observed that some plots present noisy curves, as in the case of the variations of the semi-axes ratio and the shape number. This is expected, since the change of these parameters implies a modification of the contact patch shape. Thus, this results in an adjustment of the mesh used in CONTACT which cause a non-smooth variation of the output quantities. All in all, the analysis of the variations allows to identify which are the variables that produce a more pronounced nonlinear response of the output quantities and, therefore, the ones that require a finer discretization of the LUT.

\subsection{Interpolation error in cells}

The assessment of the accuracy of the current LUT is performed by the evaluation of the interpolation error measured as the difference between the exact value of the forces and moments calculated with CONTACT and the value for the same quantities interpolated from the LUT. In order to have a proper error analysis, it is of paramount importance to select suitable points in which the error evaluation is performed. Certainly, in the LUT points the interpolation error is null as the forces and moments in these points are exact. Thus, the best candidates for points with maximum errors are those in the vicinity of the LUT cell center as shown graphically in Figure 3, for a case in which only 
3 inputs are considered. Note that in the current case there are 5 input parameters making the graphical representation of the LUT cell center impossible. Assuming that the distance between LUT points is sufficiently small to that the error introduced by the interpolation is inversely proportional to the distance to them, the point in the center of each cell would lead to the output with the highest error, since it is the one farthest from the lookup table points, for which the output is exact.

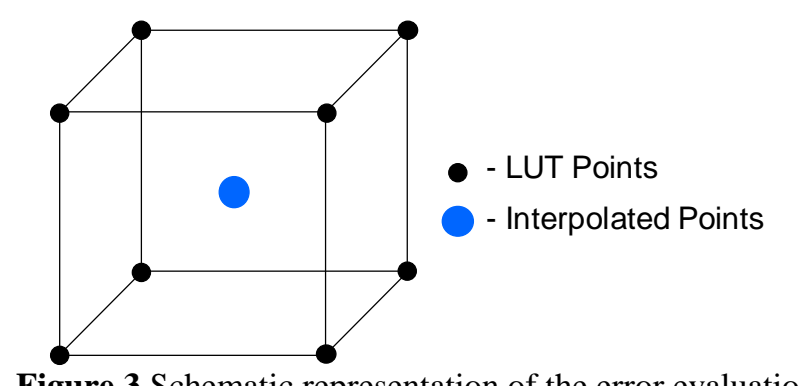

Figure 3 Schematic representation of the error evaluation at the center of a cell of a 3D lookup table.

For the LUT with the points described in Table 1, the number of cells defined in the space that it spans is

$$
N_{\mathrm{c}}=\prod_{i=1}^{5}\left(n_{i}-1\right)=64680
$$

The errors of interpolation for the center of each cell of the LUT for the forces and moment are presented in Fig. 4 for the three output quantities. The cell number, that defines the order in which each input parameter is spanned, can be evaluated as

$$
n_{c}=i_{\chi}+\left(n_{\chi}-1\right)\left(i_{g}-1+\left(n_{g}-1\right)\left(i_{\alpha}-1+\left(n_{\alpha}-1\right)\left(i_{v}-1+\left(n_{v}-1\right)\left(i_{\psi}-1\right)\right)\right)\right)
$$

where $i_{k}$ is the $i$-th space of the parameter $k$. Moreover, the interpolation error for each output quantity can be evaluated as the absolute difference between the interpolated value using LUT data and the exact value obtained with Eqs. (9)-(11), that is

$$
\begin{aligned}
& \text { Error } f_{x}\left(n_{c}\right)=\left|f_{x}\left(\chi_{c}, g_{c}, \alpha_{c}, v_{c}, \psi_{c}\right)-f_{x}^{\text {int }}\left(\chi_{c}, g_{c}, \alpha_{c}, v_{c}, \psi_{c}\right)\right| \\
& \text { Error } f_{y}\left(n_{c}\right)=\left|f_{y}\left(\chi_{c}, g_{c}, \alpha_{c}, v_{c}, \psi_{c}\right)-f_{y}^{\text {int }}\left(\chi_{c}, g_{c}, \alpha_{c}, v_{c}, \psi_{c}\right)\right| \\
& \text { Error } m_{z}\left(n_{c}\right)=\left|m_{z}\left(\chi_{c}, g_{c}, \alpha_{c}, v_{c}, \psi_{c}\right)-m_{z}^{\text {int }}\left(\chi_{c}, g_{c}, \alpha_{c}, v_{c}, \psi_{c}\right)\right|
\end{aligned}
$$

where $\chi_{c}, g_{c}, \alpha_{c}, v_{c}$, and $\psi_{c}$ are the values of the input variables in the center of the cell $n_{c}$ and $f_{x}^{\text {int }}, f_{y}^{\text {int }}$ and $m_{z}{ }^{\text {int }}$ denote the creep forces and moment obtained through linear interpolation of the LUT data.

Since all the parameters are interpolated at the same time, it is not possible to check which one is the main source of error. The results only allow to identify the order of magnitude of the maximum interpolation error of each output quantity.

\subsection{Interpolation error in edges}

In order to improve the LUT design, it is necessary to identify which are the parameters that need to be more refined and the ones that can have a coarser distribution. To address this issue, the interpolation error is evaluated at the points in the middle of the LUT edges as represented in Fig. 5. Note that when interpolating at any edge of the LUT, four input parameters are kept constant, and the interpolation is performed with the variation of a single parameter. Thus, the evaluation of the error in the middle point of 
the edges allows to determine the error introduced by the discretization of each individual input parameter.
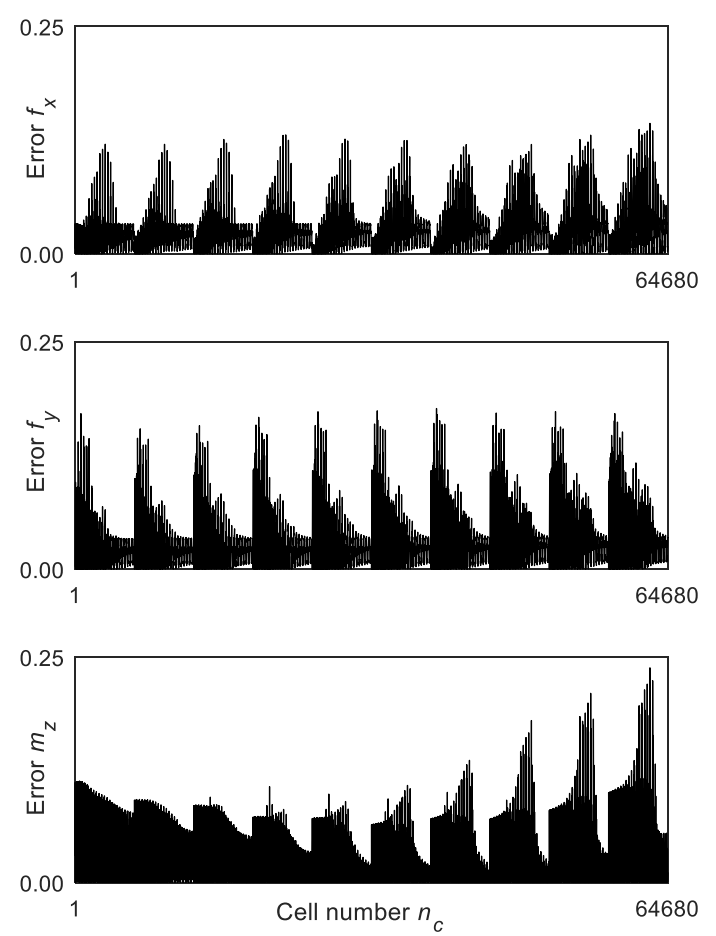

Figure 4 Interpolation error at the center of the LUT cells for different outputs over the domain.

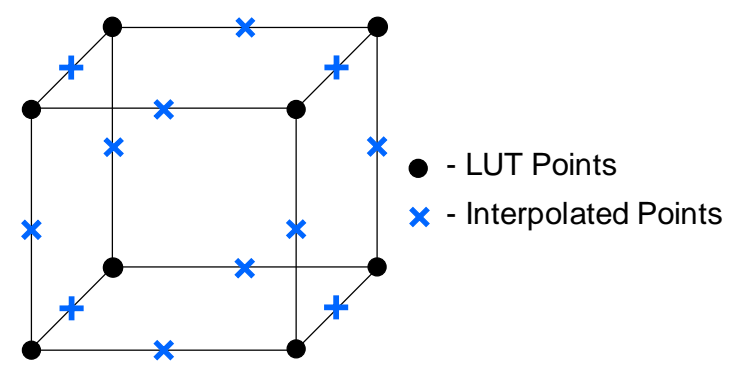

Figure 5 Schematic representation of the error evaluation at the middle point of the edges of a cell of a 3D lookup table.

The number of edges of the LUT is significantly higher than the number of cells being given, for the LUT with the structure presented in Table 1, as

$$
N_{\mathrm{e}}=\sum_{i=1}^{5} \frac{\left(n_{i}-1\right) \prod_{j=1}^{5} n_{j}}{n_{i}}=506960
$$

The maximum and mean errors of the normalized creep forces and moment are evaluated for each edge of the lookup table domain, and summarized in Figs. 6 and 7, respectively. Thus, the values of the interpolation error at the middle point of each edge are obtained with an analogous manner as for the interpolation error in the center of the cells, as it was represented by Eqs. (15)-(17). In each plot all the outputs are presented with the same scale, for easier readability and comparison.

From the analysis of the maximum interpolation errors, displayed in Fig. 6, the peak value is found for the interpolation of the spin creepage, which suggests that additional points should be used in the LUT. The higher values of spin creepages present lower 
interpolation error, actually suggesting that less points can be used in the LUT for the higher values.
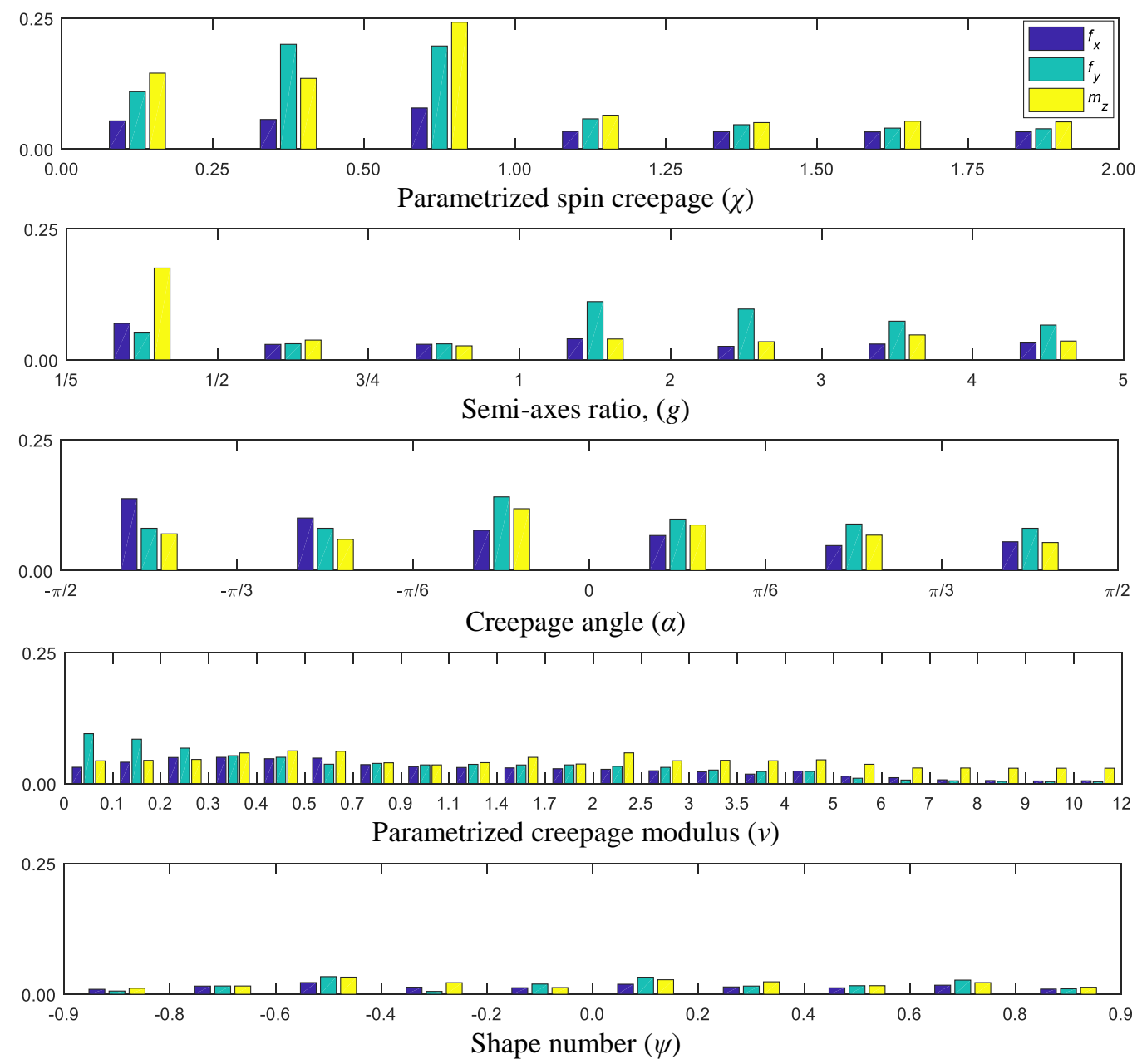

Figure 6 Maximum interpolation error between two consecutive points of the same independent parameter for each output quantity.

The semi-axis ratio is plotted in a logarithmic scale, and it shows higher errors for the larger intervals in this scale. This observation suggests that a finer refinement is required for the lower semi-axis ratio values and that a coarser refinement can be used in the higher values.

The creepage angle and modulus and the shape number have all evenly distributed errors along each domain. However, the interpolation error from the creepage modulus and the shape number present a maximum error magnitude much lower than that of the remaining parameters, which means that they can have a coarser refinement without damaging the LUT accuracy.

In what concerns to the mean error, the magnitude of error is significantly lower, as seen in Fig. 7, but a similar analysis to that made for the maximum error can be made. The maximum mean error is found to be in the lower values of the semi-axis ratio. Relatively to the remaining input variables, they have discretization which favor uniform error distributions. However, the creepage angle exhibits the highest interpolation error.

There is no advantage of having different levels of error for different input parameters, since the accuracy of the table is limited by the variable that introduces the highest error. This means that the design of the LUT can be enhanced either by improving the discretization where the error is higher, thus increasing accuracy, or by using a coarser discretization where the error is smaller, which lowers the size of the LUT. 

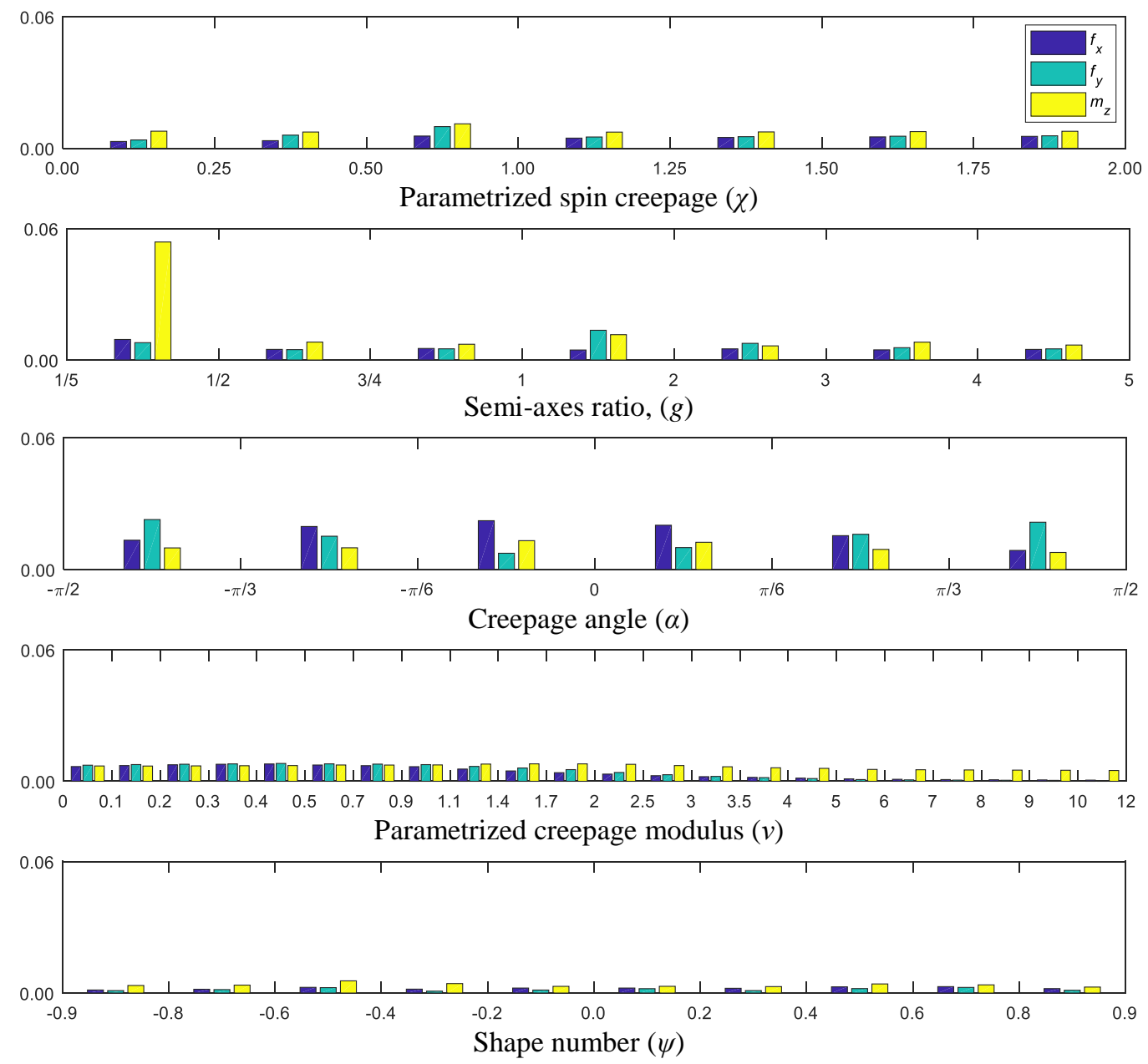

Figure 7 Mean interpolation error between two consecutive points of the same independent parameter for each output quantity.

\section{Generation of enhanced KBTNH}

As it was previously shown, the current KBTNH has an unbalanced interpolation error for the different input parameters. Based on the analysis made in the previous section, two novel LUT designs are proposed. The options are either to have a smaller sized table keeping the error level or to improve the accuracy while preserving the LUT size. A trial and error procedure, which involves the generation of table and error analysis, is used here to obtain the enhanced LUTs. For instance, the creepage angle and shape factor have the same range of errors for the positive and negative parts of their domains, therefore, they must be symmetric in this perspective. Moreover, the distribution of points of the semi-axis ratio must be symmetric about 1 in a logarithmic scale.

\subsection{Reduced Size Enhanced LUT}

In order to preserve the same accuracy of the original LUT, the parameters that lead to the higher values must be kept with the same or similar discretization in the region of such error. Thus, the creepage angle, which has an equally spaced distribution, is maintained unchanged. The spin creepage may have a coarser discretization for higher values. Regarding the semi-axis ratio, the points must be redistributed to achieve the symmetry about 1 . The number of points used for the creepage modulus and shape number can be significantly reduced, since the current error on the output quantities is much lower than for any other input parameter. 
Bearing in mind the observations on the errors due to the discretization of the input parameters, an enhanced version of the KBTNH is proposed, being its discretization described in Table 3. The number of points used for the discretization of each parameter is reduced relatively to the original LUT. The total number of data points in the enhanced LUT is now

$$
N_{p}=\prod_{i=1}^{5} n_{i}=24010
$$

Comparing the size of the enhanced LUT with the size of the original LUT, given in Eq. (12), a compression ratio of 4.72 is obtained for similar accuracy. This has a substantial impact on the time to generate the LUT and on the time to search and interpolate the LUT during dynamic analyses.

Table 3 Domain of the enhanced Kalker Book of Tables for Non-Hertzian (KBTNH) contact with reduced size.

\begin{tabular}{|c|c|c|}
\hline Parameter & No. of Points & Deviation \\
\hline$\chi=\left\{\begin{array}{llll}0.00 & 0.25 & 0.50 & 0.75 \\
1.00 & 1.50 & 2.00\end{array}\right\}$ & $n_{\chi}=7$ & -1 \\
\hline$g=\left\{\begin{array}{lllllll}\frac{1}{5} & \frac{1}{3} & \frac{1}{2} & 1 & 2 & 3 & 5\end{array}\right\}$ & $n_{\mathrm{g}}=7$ & -1 \\
\hline$\alpha=\left\{\begin{array}{rrrrrrr}-\frac{\pi}{2} & -\frac{\pi}{3} & -\frac{\pi}{6} & 0 & \frac{\pi}{6} & \frac{\pi}{3} & \frac{\pi}{2}\end{array}\right.$ & $n_{\alpha}=7$ & 0 \\
\hline$v=\left\{\begin{array}{ccccc}0.0 & 0.2 & 0.4 & 0.7 & 1.2 \\
2.0 & 3.5 & 5.0 & 8.0 & 12.0\end{array}\right\}$ & $n_{v}=10$ & -13 \\
\hline$\left.\psi=\begin{array}{llll}-0.9 & -0.6 & -0.3 \\
0.0 & 0.3 & 0.6 & 0.9\end{array}\right\}$ & $n_{\psi}=7$ & -4 \\
\hline
\end{tabular}
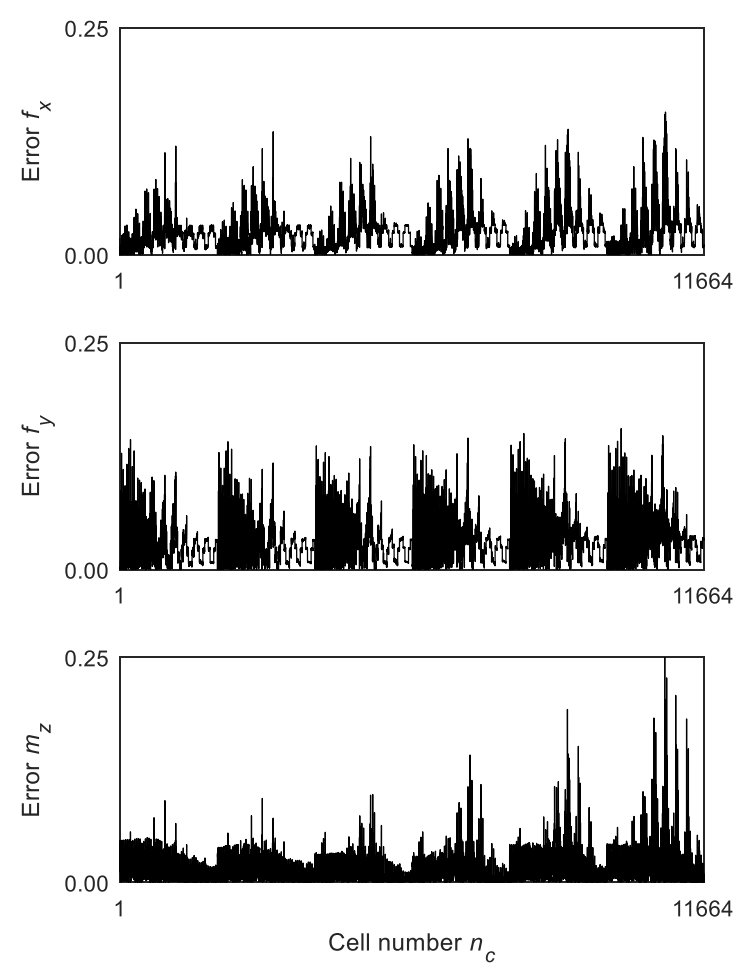

Figure 8 Interpolation error at the center of the LUT cells for different outputs over the domain of the enhanced LUT with reduced size. 
To assess the accuracy of the enhanced KBTNH and compare it with the original one, the evaluation of the interpolation error at the center of the cells and at the middle point of the edges of the LUT was performed similarly to that made in Sections 3.2 and 3.3, respectively. The error of interpolation at the center of the cells of the LUT proposed in Table 3 is displayed in Fig. 8. Moreover, Figures 9 and 10 depict, respectively, the maximum and mean errors of interpolation for the normalized creep forces and moment.

Through the comparison of the plots of Figs. 4 and 8, it can be recognized that a similar level of error in the center of the cells can be achieved with a significant reduction of the size of the LUT if a careful selection of its points is made. By observing Fig. 9 and 10 , it can be concluded that the level of maximum error is similar to the original one, i.e., lower than 0.25 . However, the maximum mean error is reduced even with the decrease of number of points used in the discretization of some input parameters. Moreover, the two parameters that are more affected by this change, i.e., the creepage modulus and shape number, keep their interpolation error lower than the creepage angle, as represented in Fig. 10.

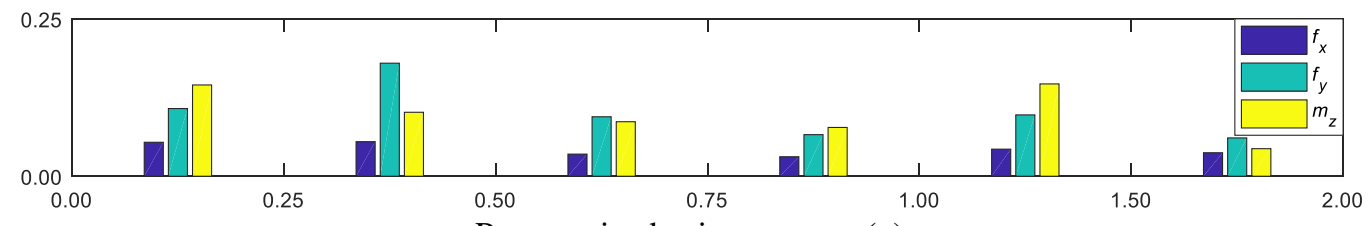

Parametrized spin creepage $(\chi)$

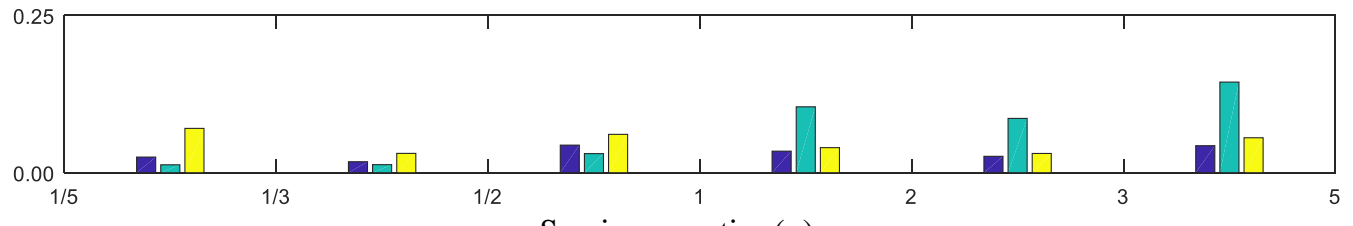

Semi-axes ratio, $(g)$
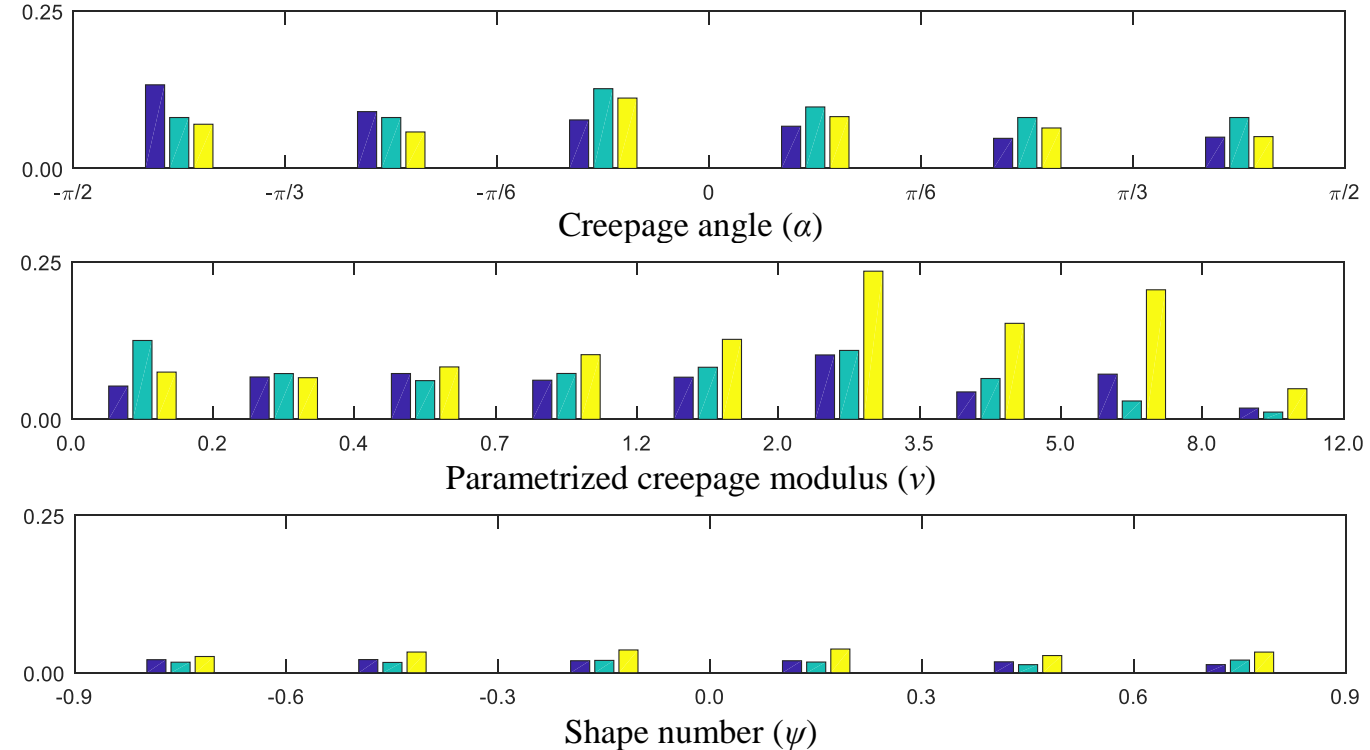

Figure 9 Maximum interpolation error between two consecutive points of the same independent parameter for each output quantity of the enhanced LUT with reduced size.

\subsection{Improved Accuracy Enhanced LUT}

By keeping the size of the LUT and redistributing the LUT points, a higher accuracy of the interpolation is obtained. Some points are removed from the zones with lower error to be placed where the error is higher. Also, the guidelines gathered from the analysis of variation of the input parameters were taken into account for the selection of LUT points. 
For this purpose, the spin creepage was refined for values between 0 and 1 to reduce the maximum interpolation error. Then, more points are added to the semi-axis ratio to maintain the same target. Since the creepage angle leads to one of the major sources of error, two additional points are considered in the discretization, keeping the constant spacing distribution. As for the enhanced LUT presented in section 4.1, the number of points for the creepage modulus and shape factor can be reduced due to original low interpolation error associated to them.
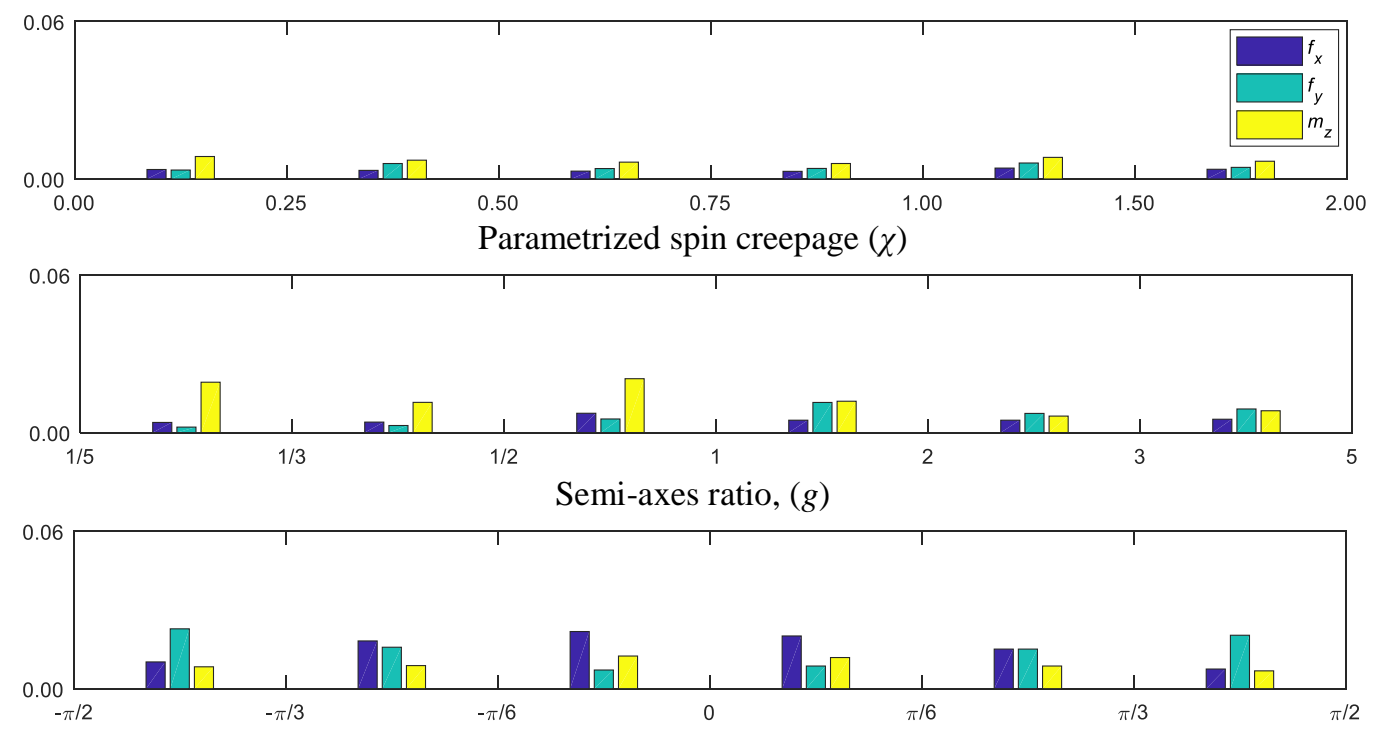

Creepage angle $(\alpha)$
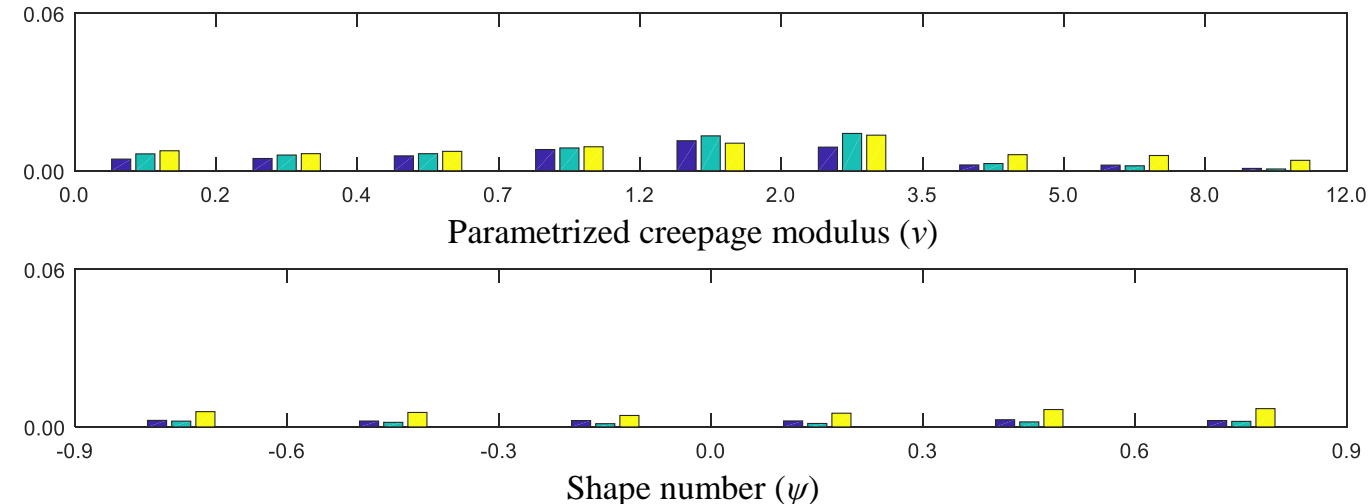

Figure 10 Mean interpolation error between two consecutive points of the same independent parameter for each output quantity of the enhanced LUT with reduced size.

Based on the observations made, an enhanced KBTNH described in Table 4 is proposed. The number of points used in the discretization is increased for the spin creepage, semi-axis ratio and creepage angle, while it is decreased for the discretization of the creepage modulus and shape number. The total number of LUT entries is now

$$
N_{p}=\prod_{i=1}^{5} n_{i}=117117
$$

The new enhanced LUT is, approximately, 1.03 times bigger than the original KBTNH, which is acceptable. To verify the accuracy enhancement of this table, the interpolation error at the center of the LUT cells is evaluated, and its values are presented in Fig. 11. From its analysis, it can be demonstrated that a significant reduction of the error has been accomplished comparatively to the original LUT. Also, the assessment of the interpolation error at the middle point of LUT edges is performed, being the maximum and mean interpolation errors for the normalized creep forces and moment shown in Fig. 
12 and 13, respectively. The results indicate that the maximum error is below 0.12 which is less than half of that exhibited by the original KBTNH. It can be recognized that, for this enhanced LUT, the interpolation error is much more balanced than in the original one, i.e., the order of magnitude of the error is similar for the five parameters. Moreover, this large decrease of the error provides higher confidence for the use of this new LUT on railway vehicle dynamics, since it has higher resemblance with the online use of CONTACT, deemed as the correct representation of the rail-wheel contact forces.
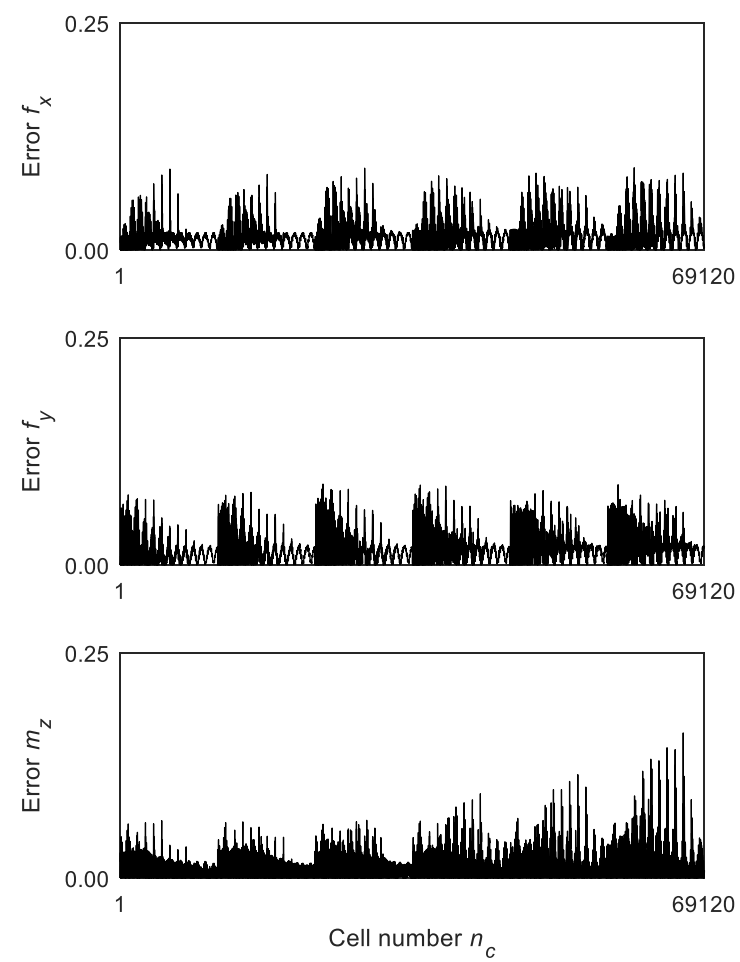

Figure 11 Interpolation error at the center of the LUT cells for different outputs over the domain of the enhanced LUT with improved accuracy.

Table 4 Domain of the enhanced Kalker Book of Tables for Non-Hertzian (KBTNH) contact with improved accuracy.

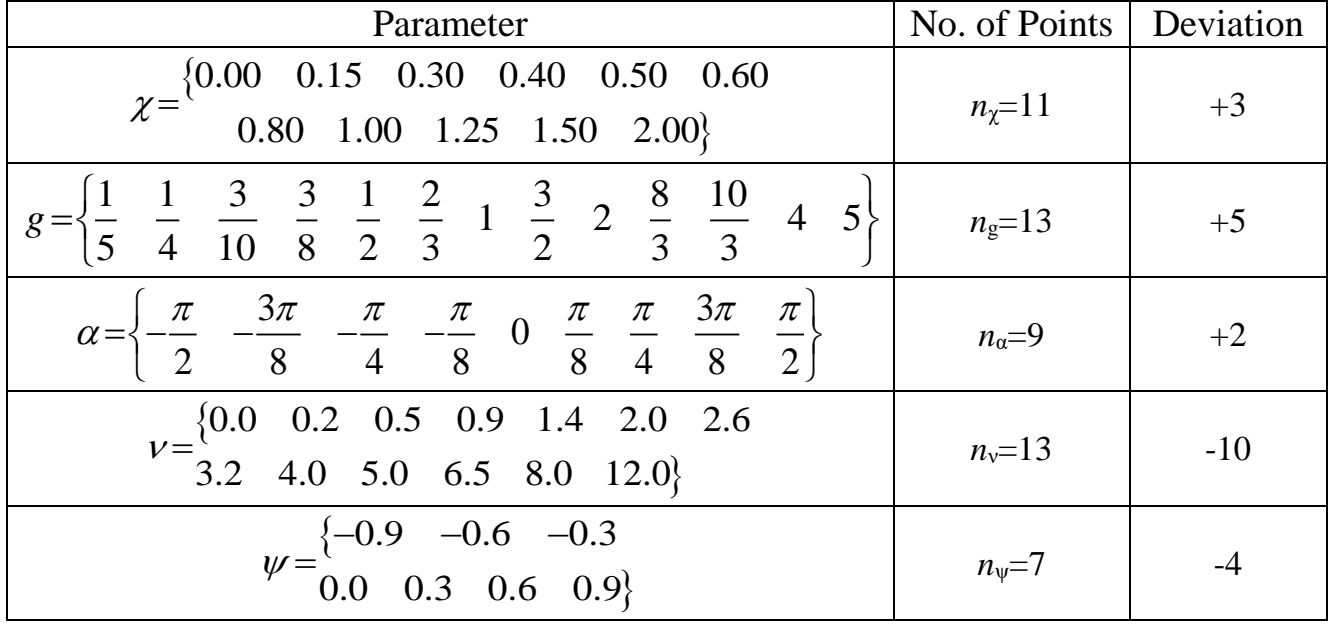



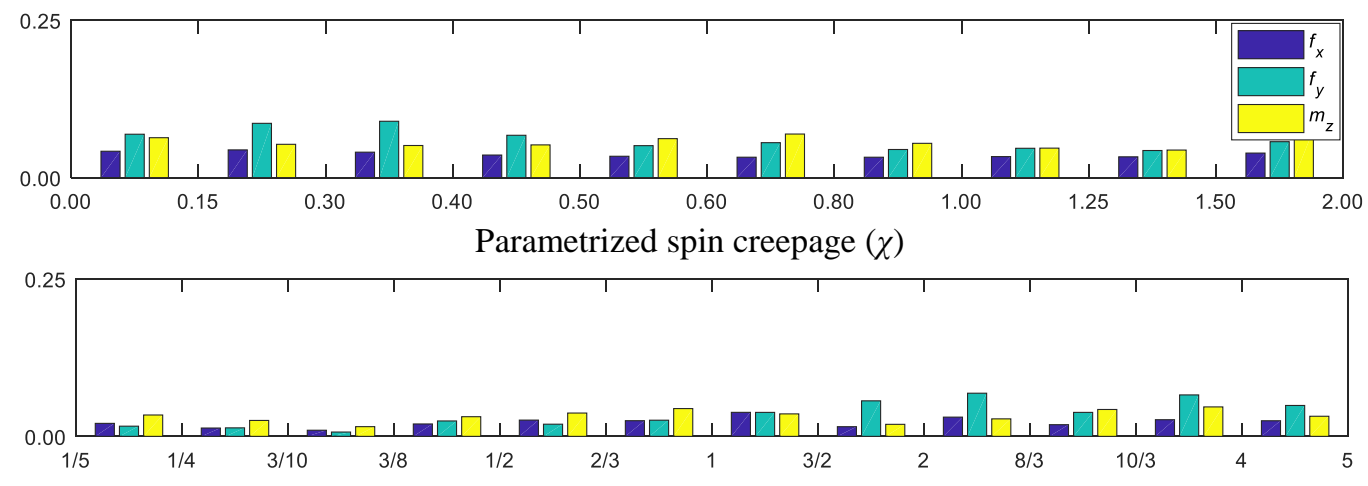

Semi-axes ratio, $(g)$

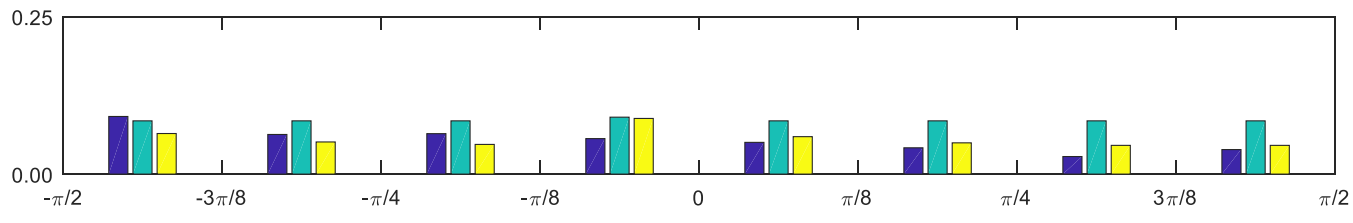

Creepage angle $(\alpha)$
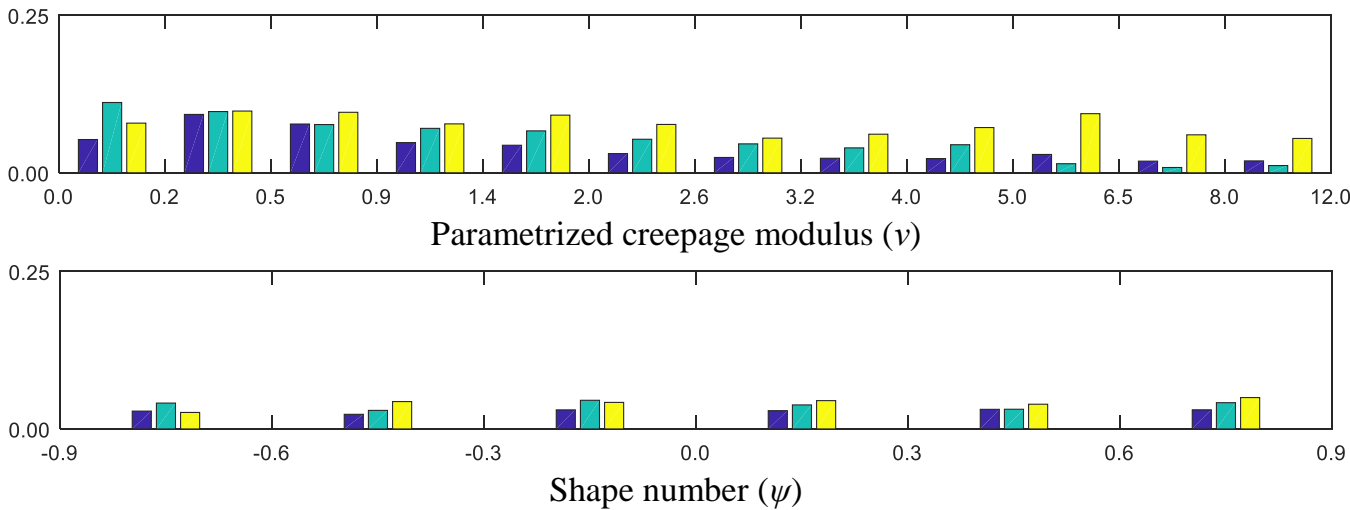

Figure 12 Maximum interpolation error between two consecutive points of the same independent parameter for each output quantity of the enhanced LUT with improved accuracy.

\section{Conclusions}

The improvement of efficiency and accuracy in railway simulations demands the development of advanced computational tools. This works presented the analysis and improvement of the KBTNH utilized for the calculation of creep forces and moment in the wheel-rail interaction. Firstly, the original LUT was characterized, and the assessment of its accuracy was performed through an analysis of the variation of its input parameters and an interpolation error evaluation. This analysis showed that the interpolation error introduced by the distribution of the LUT points was significantly different for the several input parameters. This observation allowed to redistribute the location of the LUT points, and even eliminate some of them where the LUT was too refined. Based on this error analysis methodology, two enhanced LUTs were generated, one with a reduced size but similar accuracy to the original and another with the same size of the original LUT but with improved accuracy. The former, with similar accuracy, has its size decreased by a ratio of 4.72. The latter, with a size identical to that of the original, exhibits a maximum interpolation error reduced by half. In view of the current computational capacity, the strategy for reducing the interpolation error seems more logical, although the decrease of the size of LUT can be preponderant for problems with even more input parameters.

The methodology presented to perform the accuracy assessment of the KBNHT can be employed in other types of LUTs and support the generation of a more accurate LUT with evenly distributed errors. Further studies may consist of analyzing the impact of 
using a more accurate table on the dynamic simulation of railway vehicles and developing an optimization technique to generate automatically LUTs with prescribed accuracy and minimal size.
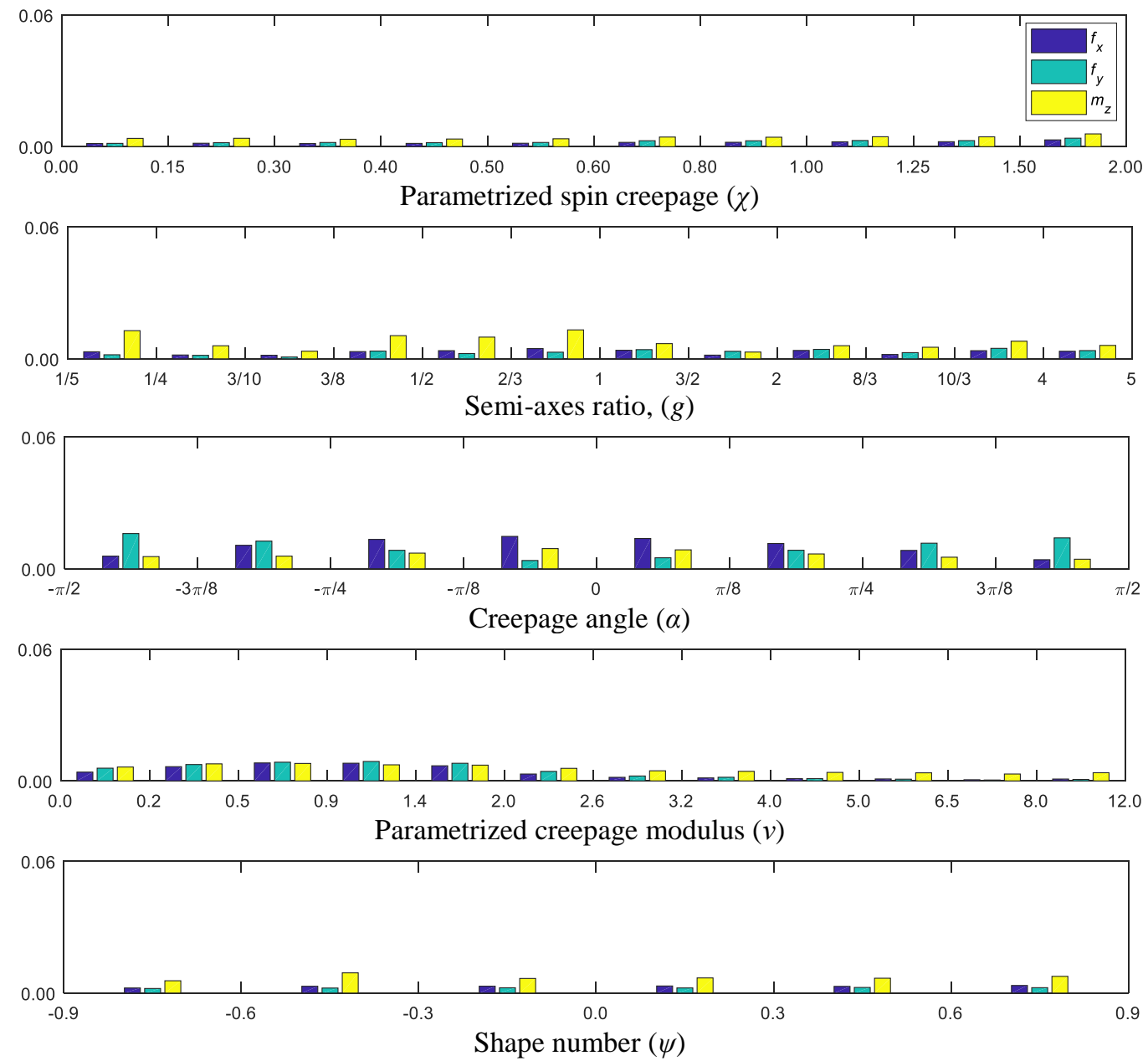

Figure 13 Mean interpolation error between two consecutive points of the same independent parameter for each output quantity of the enhanced LUT with improved accuracy.

\section{Acknowledgments}

The first and second authors are supported by the Portuguese Foundation for Science and Technology (FCT) under grant PD/BD/114154/2016, MIT Portugal Program, and SFRH/BD/96695/2013, respectively. This work is supported by FCT with the reference project UID/EEA/04436/2019.

\section{References}

[1] W. Kortüm: Review of Multibody Computer Codes for Vehicle System Dynamics, Vehicle System Dynamics, 1993, 22(S1), 3-31.

[2] S. Alfi and S. Bruni: Mathematical modelling of train-turnout interaction, Vehicle System Dynamics, 2009, 47(5), 551-574.

[3] M.S. Sichani, R. Enblom and M. Berg: Non-Elliptic Wheel-Rail Contact Modelling in Vehicle Dynamics Simulation, International Journal of Railway Technology, 2014, 3(3), 7796.

[4] G. Götz and O. Polach: Influence of varying the input parameters on the results of model validation, Proceedings of the Institution of Mechanical Engineers, Part F: Journal of Rail and Rapid Transit, 2017, 231(5), 598-609.

[5] P. Antunes, H. Magalhães, J. Ambrósio, J. Pombo and J. Costa: A co-simulation approach 
to the wheel-rail contact with flexible railway track, Multibody System Dynamics, 2019, 45(2), 245-272.

[6] A. Rovira, A. Roda, M.B. Marshall, H. Brunskill, and R. Lewis: Experimental and numerical modelling of wheel-rail contact and wear, Wear, 2011, 271, 911-924.

[7] J. Pombo, J. Ambrósio, M. Pereira, R. Lewis, R. Dwyer-Joyce, C. Ariaudo, and N. Kuka: Development of a Wear Prediction Tool for Steel Railway Wheels Using Three Alternative Wear Functions, Wear, 2011, 271, 238-245.

[8] G. Tao, D. Ren, L. Wang, Z. Wen and X. Jin: Online prediction model for wheel wear considering track flexibility, Multibody System Dynamics, 2018, 44(3), 313-334.

[9] R. Conti, E. Galardi, E. Meli, D. Nocciolini, L. Pugi and A. Rindi: Energy and wear optimisation of train longitudinal dynamics and of traction and braking systems, Vehicle System Dynamics, 2015, 53(5), 651-671.

[10] H. Magalhães, J.F.A. Madeira, J. Ambrósio and J. Pombo: Railway vehicle performance optimisation using virtual homologation, Vehicle System Dynamics, 2016, 54(9), 1177-1207

[11] J.J. Kalker: Three-dimensional elastic bodies in rolling contact. Kluwer Academic Publishers, 1990.

[12] J. Pombo, J. Ambrósio and M. Silva: A New Wheel-Rail Contact Model for Railway Dynamics, Vehicle System Dynamics, 2007, 45(2), 165-189.

[13] J. Piotrowski and W. Kik: A simplified model of wheel/rail contact mechanics for nonHertzian problems and its application in rail vehicle dynamic simulations, Vehicle System Dynamics, 2008; 46(1,2) 27-48.

[14] A. Alonso, A. Guiral and J.G. Gimenez: Wheel Rail Contact: Theoretical and Experimental Analysis, International Journal of Railway Technology, 2013, 2(4), 15-32

[15] M.S. Sichani, R. Enblom and M. Berg: A novel method to model wheel-rail normal contact in vehicle dynamic simulation, Vehicle System Dynamics, 2014, 52(12) 1752-1764

[16] B. Liu, S. Bruni and E. Vollebregt: A non-Hertzian method for solving wheel-rail normal contact problem taking into account the effect of yaw, Vehicle System Dynamics, 2016, 54(9) 1226-1246.

[17] N. Burgelman, M.S. Sichani, R. Enblom, M. Berg, Z. Li and R. Dollevoet: Influence of wheel-rail contact modelling on vehicle dynamic simulation, Vehicle System Dynamics, 2015, 53(8) 1190-1203.

[18] J.J. Kalker: Introduction to the Fortran IV Program DUVOROL and CONTACT for the Solution of 3D Elastostatic Half-Space Contact Problems with and without Friction.

Technical Report, Delft University of Technology, Netherlands, 1982.

[19] J.J. Kalker: On the Rolling Contact of Two Elastic Bodies in the Presence of Dry Friction. $\mathrm{PhD}$ Thesis, Delft University of Technology, Netherlands, 1967.

[20] J.J. Kalker: A fast algorithm for the simplified theory of rolling contact, Vehicle System Dynamics, 1982, 11, 1-13.

[21] O. Polach: A Fast Wheel-Rail Forces Calculation Computer Code, Vehicle System Dynamics, 1999, Sup. 33, 728-739.

[22] E.A.H. Vollebregt and P. Wilders: FASTSIM2: a second-order accurate frictional rolling contact algorithm, Computational Mechanics, 2011, 47(1), 105-116.

[23] Z.Y. Shen, J.K. Hedrick and J.A. Elkins: A Comparison of Alternative Creep Force Models for Rail Vehicle Dynamic Analysis, Vehicle System Dynamics, 1983, 12(1-3), 79-83.

[24] A. Palermo, D. Mundo, R. Hadjit and W. Desmet: Multibody element for spur and helical gear meshing based on detailed three-dimensional contact calculations, Mechanism and Machine Theory, 2013, 62, 13-30.

[25] H. Li, A. Terao and H. Sugiyama: Application of tabular contact search method to multibody gear dynamics simulation with tooth surface imperfections, Proceedings of the Institution of Mechanical Engineers, Part K: Journal of Multi-body Dynamics, 2014, 229(3), 274-290.

[26] M. Machado, P. Flores and J. Ambrósio: A Lookup-Table-Based Approach for Spatial Analysis of Contact Problems, Journal of Computational and Nonlinear Dynamics, 2014, 9(4), 041010.

[27] M. Bozzone, E. Pennestrì, and P. Salvini: A lookup table-based method for wheel-rail 
contact analysis, Proceedings of the Institution of Mechanical Engineers, Part K: Journal of Multi-body Dynamics, 2011 225(2), 127-138.

[28] J.L. Escalona and J.F. Aceituno: Multibody simulation of railway vehicles with contact lookup tables, International Journal of Mechanical Sciences, 2018, https://doi.org/10.1016/j.ijmecsci.2018.01.020

[29] J. Kalker, Book of tables for the Hertzian creep-force law, in Proceedings of the 2nd Mini Conference on Contact mechanics and Wear of Wheel/Rail Systems, I. Zobory, ed., Budapest University of Technology and Economics, Budapest, 1996, 11-20.

[30] J. Piotrowski, B. Liu and S. Bruni: The Kalker book of tables for non-Hertzian contact of wheel and rail, Vehicle System Dynamics, 2017, 55(6) 875-901.

[31] K. Rose: Kalker Table for Wheel/rail Force Prediction, Internal Memorandum IM VTI 4, British Rail Research, Derby, UK, 1984.

[32] E.A.H. Vollebregt: Refinement of Kalker's rolling contact model, In A. Bracciali, editor, Proceedings of the 8th International Conference on Contact Mechanics and Wear in Rail/Wheel Systems, 149-156, 2009.

[33] J. Piotrowski, S. Bruni, B. Liu and E. Di Gialleonardo: A fast method for determination of creep forces in non-Hertzian contact of wheel and rail based on a book of tables, Multibody System Dynamics, 2018, https://doi.org/10.1007/s11044-018-09635-3

[34] E.A.H. Vollebregt, S.D. Iwnicki, G. Xie, and P. Shackleton: Assessing the accuracy of different simplified frictional rolling contact algorithms, Vehicle System Dynamics, 2012, $50(1), 1-17$.

[35] H.M. Aus and G.A. Korn: Table-Lookup/Interpolation Function Generation for FixedPoint Digital Computations, IEEE Transactions on Computers, 1969, C-18(8), 745-749.

[36] L. Deng, C. Chakrabarti, N. Pitsianis and X. Sun: Automated optimization of look-up table implementation for function evaluation on FPGAs, Proceedings of SPIE - The International Society for Optical Engineering, 2009, no. 744413.

[37] C. Wilcox, M.M. Strout, and J.M. Bieman: Mesa: Automatic generation of lookup table optimizations, Proceedings - International Conference on Software Engineering, 2011, 1-8

[38] H. Magalhães, F. Marques, B. Liu, J. Pombo, P. Flores, J. Ambrósio and S. Bruni: An Optimization Approach to Generate Accurate and Efficient Lookup Tables for Engineering Applications, 6th International Conference on Engineering Optimization - EngOpt 2018, Lisbon, Portugal, September 17-19, 2018, 12 pages.

[39] R.H. Myers, D.C. Montgomery and C.M. Anderson-Cook: Response Surface Methodology: Process and Product Optimization Using Designed Experiments, Fourth Edition, John Wiley \& Sons, Inc., New York, New York, 2016. 